\title{
Anti-TRAPISSP2 monoclonal antibodies can inhibit sporozoite infection and enhance protection of anti-CSP monoclonal antibodies
}

Brandon K. Wilder*1†\#, Vladimir Vigdorovich*1, Sara Carbonetti ${ }^{1}$, Nana Minkah ${ }^{1}$, Nina Hertoghs ${ }^{1}$, Andrew Raappana ${ }^{1}$, Hayley Cardamone ${ }^{1}$, Brian G. Oliver ${ }^{1}$, Olesya

Trakhimets $^{1}$, Sudhir Kumar ${ }^{1}$, Nicholas Dambrauskas ${ }^{1}$, Silvia A. Arredondo ${ }^{1}$, Nelly

Camargo $^{1}$, Stefan H.I. Kappe ${ }^{\star 1,2,3}$ and D. Noah Sather*1,2,3

${ }^{1}$ Center for Global Infectious Disease Research, Seattle Children's Research Institute, Seattle, WA, USA

${ }^{2}$ Department of Pediatrics, University of Washington, Seattle, WA, USA

${ }^{3}$ Department of Global Health, University of Washington, Seattle, WA, USA

†Present address: Vaccine and Gene Therapy Institute, Oregon Health \& Science

University, Beaverton, Oregon, 97006, USA

${ }^{*}$ Equal contribution

Correspondence: D. Noah Sather: noah.sather@seattlechildrens.org and

Stefan H.I. Kappe: stefan.kappe@seattlechildrens.org 


\section{Summary}

2 Vaccine-induced sterilizing protection from infection with the Plasmodium parasite, the

3 pathogen that causes malaria, will be an essential tool in the fight against malaria as it

4 would prevent both malaria-related disease and transmission. Stopping the relatively

5 small number of parasites injected by the mosquito before they can migrate from the

6 skin to the liver is an attractive goal. Antibody-eliciting vaccines have been used to

7 pursue this objective by targeting the major parasite surface protein present during this

8 stage, the circumsporozoite protein (CSP). While CSP-based vaccines have recently

9 had encouraging success in disease reduction, this was only achieved with extremely

10 high antibody titers and appeared less effective for a complete block of infection. While

11 such disease reduction is important, these results also indicate that further

12 improvements to vaccines based solely on CSP will likely yield diminishing benefits

13 towards the goal of durable, infection-blocking immunity. Here, we show that

14 monoclonal antibodies (mAbs) recognizing the sporozoite protein TRAP/SSP2 across

15 the major protein domains exhibit a range of inhibitory capacity and that these mAbs

16 can augment CSP-based protection despite delivering no sterile protection on their own.

17 Therefore, pursuing a multivalent subunit vaccine immunization is a promising strategy

18 for improving infection-blocking malaria vaccines. 


\section{Introduction}

2 The last four years have marked a disheartening milestone as the first time in a

3 generation without a reduction in the global burden of malaria (WHO) ${ }^{1}$. The

4 interventions that have provided much of the previous progress, such as insecticide-

5 treated bednets and large scale treatment programs, are highly susceptible to

6 interruptions due to political or economic instability. This was starkly illustrated by the

7 resurgence of malaria in Venezuela after near-elimination and also as predicted

8 following the COVID-19 pandemic ${ }^{1}$. Therefore, it is likely that long-lasting, infection-

9 blocking interventions (e.g. vaccines, long-lasting monoclonal antibodies or

10 chemoprophylactics) will be required to drive malaria to elimination.

12 Developing such an intervention is hampered by the complex life cycle of the parasite,

13 which begins when an infected mosquito injects tens to hundreds of the "sporozoite"

14 forms of the parasite into the dermis ${ }^{2}$. From here, sporozoites actively traverse through

15 multiple cell types in search of an endothelial cell through which they will gain access to

16 the blood ${ }^{3}$. They are carried to the liver within minutes, where they traverse multiple cell

17 types in the liver parenchyma and eventually establish infection in a hepatocyte ${ }^{4}$.

18 Following $\sim 7-10$ days of asexual replication and transformation ( $\sim 2$ days in rodent

19 malaria models), each successful liver stage releases $30,000-50,000$ "merozoites" that

20 cyclically infect, replicate within and lyse red blood cells ${ }^{5,6}$. It is only during this blood

21 stage of infection when symptomatic disease occurs and is also where a subset of

22 sexually differentiated parasite forms can be picked up by a new mosquito host to

23 continue the transmission cycle. Each step in the infection cycle presents an opportunity 
1 for intervention, although vaccines targeting the "pre-erythrocytic" stages in the skin and

2 liver have yielded the most promising results ${ }^{7}$.

4 The most advanced pre-erythrocytic vaccine is RTS,S-an antibody-eliciting subunit

5 vaccine targeting the major sporozoite surface protein circumsporozoite protein (CSP) ${ }^{8}$.

6 Vaccines based on attenuated live sporozoites that arrest in the liver and function by a

7 combination of T cells and antibodies have also demonstrated robust protection ${ }^{9}$.

8 Unfortunately, despite significant efficacy from both approaches in controlled human

9 malaria infection (CHMI) studies in malaria-naïve volunteers, both vaccines have

10 markedly reduced efficacy in field trials and have not met the goals of $75-80 \%$

11 protection for vaccines ${ }^{10}$ or injectable anti-malarials ${ }^{11}$. Recent encouraging Phase II

12 results with the R21 CSP particle in Matrix-M adjuvant do meet the WHO goal of $75 \%$

13 protection against clinical disease for one year ${ }^{12}$. However, this protection appears less

14 robust in preventing infection and depends on high antibody titers requiring yearly

15 boosters that are vulnerable to the same interruptions as current interventions. These

16 realities highlight the significant room for improvement in both $\mathrm{T}$ cell and antibody-

17 eliciting vaccines, with the latter more amenable to iterative improvement due to

18 available in vitro and in vivo preclinical assays ${ }^{13-16}$.

20 Of the hundreds of proteins expressed at the sporozoite stage, at least 47 are surface-

21 exposed ${ }^{17-19}$ and therefore potentially accessible to antibodies. However, few of these

22 proteins have been rigorously investigated for their use in antibody-eliciting vaccines ${ }^{20}$.

23 In addition to CSP, the thrombospondin-related anonymous protein (TRAP, also known 
1 as sporozoite surface protein 2 or SSP2) has been pursued as a vaccine candidate due

2 to its high abundance, essentiality for sporozoite infection and correlation with protection

3 in naturally-immune persons ${ }^{21,22}$. The TRAP ectodomain consists of 3 main domains: a

4 von Willebrand factor A-like domain (vWA), the thrombospondin repeat (TSR) domain

5 and a repeat domain ${ }^{21}$. The most advanced TRAP vaccine candidate is an

6 adenovirus/MVA-vectored vaccine eliciting strong $\mathrm{T}$ cell responses that has had low or

7 mixed efficacy results in CHMI trials ${ }^{23,24}$ and field trials ${ }^{25}$ but has been improved in

8 mice following targeting of the $\mathrm{T}$ cell response to the liver ${ }^{26}$. A combination protein

9 TRAP/RTS,S immunization failed to show significant protection ${ }^{27}$, while a fusion-protein

10 approach using TRAP and CSP resulted in complete protection ${ }^{28}$. Antibody function in

11 experiments involving immunization with TRAP-derived peptides yielded mixed results

12 ranging from significant sporozoite inhibition in vitro ${ }^{29}$ to no protection in vivo ${ }^{30}$. In

13 summary, the results using TRAP alone or in combination with CSP are difficult to

14 interpret due to the diversity of vaccine platforms used, the possibility of immune

15 interference in studies combining platforms, and the unclear dominance of roles for

16 antibodies and $\mathrm{T}$ cells in protection ${ }^{31}$. Whether a more targeted TRAP antibody

17 response could contribute to protection either alone or in combination with CSP remains

18 poorly defined.

20 Here, we used both active immunization and passive transfer of monoclonal antibodies

21 raised against either Plasmodium yoelii (rodent malaria) and Plasmodium falciparum

22 (human malaria) TRAP to more directly explore the potential efficacy of anti-TRAP

23 antibodies. We found that anti-TRAP antibodies modestly prevent liver infection in a 
1 manner dependent on the TRAP domain recognized. Importantly, however, we

2 demonstrate proof of concept that anti-TRAP antibodies with minimal protective

3 capacity of their own can augment anti-CSP antibodies, providing additive protection

4 that raises their protective efficacy above the desired threshold of $75-80 \%$ protection.

5 Together, these findings argue for further investigation of rationally designed antibody-

6 eliciting malaria vaccines that targets multiple antigens and might include CSP as well

7 as non-CSP targets such as TRAP.

10 Results

11 PYTRAP polyclonal antibodies can prevent parasite infection of hepatocytes in vitro and

12 in vivo.

13 To elicit potentially functional anti-TRAP antibodies, we generated full-length

14 ectodomains and fragments of both rodent ( $P$. yoelii) and human $(P$. falciparum) malaria

15 TRAP proteins (Fig. 1A; Suppl. Table 1) and verified their purity (Fig. 1B). Serum from

16 mice immunized with the rodent malaria $P$. yoelii TRAP ectodomain (PyTRAP)

17 recognized Py sporozoites by immunofluorescence in a pattern consistent with

18 micronemal localization, indicating the antigenic fidelity of the recombinant protein (Fig.

19 2A). We further tested this serum in an inhibition of sporozoite traversal and invasion

20 (ISTI) assay. Compared to control serum, anti-PyTRAP serum was able to modestly but

21 significantly $(p=0.028)$ reduce sporozoite invasion of Hepa1-6 hepatoma cells in vitro at

22 a level similar to serum from mice immunized with the recombinant PyCSP ectodomain,

23 although the latter failed to reach significance $(p=0.106)$ (Fig. 2B). In contrast, 
1 sporozoite traversal of Hepa1-6 cells was not affected by anti-PyTRAP serum

$2(p=0.125)$, whereas anti-PyCSP serum did significantly reduce traversal $(p=0.0057)$

3 (Fig. 2C). The known inhibitory anti-PyCSP mAb 2F6 reduced both inhibition and

4 traversal in this assay as expected (Fig. 2B and C).

6 PyTRAP-immunized mice were then challenged with Py sporozoites via mosquito bite to 7 determine if these antibodies could function in vivo to reduce liver infection. We utilized

8 a PyGFPluc parasite, which expresses luciferase, enabling the measurement of liver

9 stage parasite burden by bioluminescence imaging. In contrast to mice immunized with

10 a non-specific control protein (Env), which showed no reduction in parasite liver stage

11 burden following challenge compared with naive mice, mice immunized with the

12 PyTRAP ectodomain demonstrated a significant $62 \%$ reduction of parasite liver stage

13 burden. Mice immunized with PyCSP ectodomain had a 91\% reduction relative to naive

14 controls (Fig. 2D). Together, these data indicate that anti-PyTRAP antibodies can

15 function in vitro and in vivo to reduce parasite infection of hepatocytes.

17 PYTRAP monoclonal antibodies display a diverse array of functions in vitro and can

18 provide additive protection to anti-CSP antibodies in vivo.

19 Serum polyclonal antibodies, as studied above, are a mixture of many antibody

20 specificities, making it difficult to characterize the relative contribution to functional

21 activity of responses directed at different domains. To enable such a characterization of

22 the repertoire of PyTRAP-elicited antibodies, we produced a panel of $15 \mathrm{mAbs}$. When

23 tested in ISTI at 10, 50 and $100 \mu \mathrm{g} / \mathrm{mL}, 12$ of these mAbs significantly inhibited invasion 
1 or traversal at one or more concentrations, with mAbs TY03 and TY11 showing the

2 most consistent and potent inhibition (Fig. 3A, Suppl. Fig. 1).

4 Overall, the mAbs demonstrated a wide range of binding affinities to recombinant

5 PyTRAP (Fig. 3B, Suppl. Table 2) and recognized epitopes in the vWA, TSR and

6 repeat regions (Suppl. Table 3), thus covering the entire protein ectodomain. Among

7 the 15 mAbs recovered, 10 mAbs bound to the vWA domain. Six of these (TY02, TY05,

8 TY06, TY10, TY11, TY20) shared variable-segment assignments for both heavy and

9 light chains, had closely related complementarity-determining-region (CDR) sequences

10 and had $88.4-96.7 \%$ and $93.9-96.9 \%$ sequence identity in the variable-region

11 sequences of their heavy and light chain, respectively (Suppl. Table 4 and Suppl. Fig.

12 2). As expected, these antibodies were functionally similar in that they bound

13 specifically to the vWA domain (Suppl. Table 3) and inhibited sporozoite infection in

14 vitro (Fig. 3A, Suppl. Fig. 1). Two mAbs specifically recognized the TSR domain, and

15 the remaining three mAbs bound epitopes in the repeat region (Suppl. Table 3). These

16 non-vWA antibodies had only modest or no sporozoite inhibition of infection in vitro (Fig.

17 3A). This panel of mAbs also showed a wide range of binding kinetics, with all strongly

18 inhibitory mAbs having a $k_{o n}$ of $>10^{5} \mathrm{M}^{-1} \mathrm{~s}^{-1}$ and a $k_{\text {dis }}$ of $<10^{-2} \mathrm{~s}^{-1}$ (Figure $3 \mathrm{~B}$, note the

19 red box, Suppl. Table 4). Together, these data demonstrate that, similar to polyclonal

20 antibodies, anti-PyTRAP mAbs can mediate anti-parasitic function in vitro, and that

21 inhibitory function likely depends on fast and stable binding to the vWA domain,

22 although within the vWA domain there might exist epitopes that show higher

23 vulnerability to blocking of infection. 
2 We next wanted to determine whether an anti-PyTRAP mAb could provide sterilizing

3 protection in vivo on its own or in combination with an anti-CSP mAb. For this, we chose

4 three vWA domain-binding anti-PyTRAP mAbs: TY03 and TY11, which were the top-

5 performing mAbs in ISTI, and TY12, which failed to demonstrate efficacy in ISTI. The

6 anti-PyTRAP mAbs were given at $300 \mu \mathrm{g} / \mathrm{mouse}(\sim 15 \mathrm{mg} / \mathrm{kg})$ alone or with a partially

7 protective dose of $100 \mu \mathrm{g} /$ mouse $(\sim 5 \mathrm{mg} / \mathrm{kg})$ of anti-PyCSP mAb 2F6 prior to mosquito

8 bite challenge ${ }^{32}$. As shown in Fig. $3 \mathrm{C}$ and Table 1, mice administered anti-PyCSP mAb

9 2F6 showed significant sterile protection, with 9/18 (50\%) remaining parasite-free,

10 compared to $2 / 15(13.3 \%)$ for mice receiving non-specific murine $\lg G(p=0.032)$. Neither

11 TY11 nor TY12 showed any protection (2/13 or 15.4\% non-infected) despite TY11

12 demonstrating the most robust inhibition in vitro. Administration of the mAb TY03

13 resulted in 7/16 mice (43.7\%) remaining parasite-free which did not reach significance

$14(p=0.072)$. When combined with the anti-CSP mAb, only the addition of TY03 afforded

15 significant sterile protection $(87.5 \%$ or $14 / 16$ mice) over the control group $(p<0.001)$

16 which, importantly, was a significant improvement over protection observed with anti-

17 PyCSP mAb alone $(p=0.025)$. Together these data indicate that while in vitro testing of

18 mAbs can be useful for identifying non-functional mAbs (e.g. TY12), they should be

19 validated in vivo for function. Importantly, these data provide proof of concept that non-

20 CSP antibodies can provide at least additive protection to anti-CSP antibodies, even

21 when those non-CSP antibodies only provide partial levels of protection on their own. 
1 Antibodies targeting the human malaria parasite $P$. falciparum TRAP can function

2 against sporozoite invasion of hepatocytes.

3 We next wanted to determine if antibodies directed against TRAP/SSP2 from the

4 human malaria parasite, $P$. falciparum, could also function to prevent sporozoite

5 infection. Serum from mice immunized with the ectodomain of $P$. falciparum TRAP

6 (PfTRAP) was able to recognize Pf sporozoites in IFA (Fig. 4A) and demonstrated

7 consistent inhibition of $P f$ sporozoite invasion in vitro at a level similar to serum from

8 mice immunized with the ectodomain of $P$. falciparum CSP (PfCSP) (Fig. 4B). Inhibition

9 of sporozoite traversal in vitro was more modest as compared to anti-PfCSP polyclonal

10 serum (Fig. 4C). The known inhibitory anti-PfCSP mAb 2 A10 demonstrated robust

11 inhibition of both invasion and traversal (Fig. 4A and B).

13 Using a similar approach to the anti-PyTRAP work described above, we isolated 7 anti-

14 PfTRAP mAbs from immunized mice. Of these mAbs, 5 recognized the vWA domain

15 and 2 recognized the TSR domains (Suppl. Table 3). In contrast to the high proportion

16 of functional anti-PyTRAP mAbs (12 of 15), only 2 of 7 anti-PfTRAP mAbs, both

17 recognizing the vWA domain, showed any sporozoite-inhibitory function in vitro: AKBR-

184 and AKBR-10. Further, only AKBR-4 demonstrated significant inhibition of both

19 invasion and traversal (Fig. 5A), despite having unremarkable binding properties with

20 the PfTRAP ectodomain (Fig. 5B). Surprisingly, mAb AKBR-7, which had the best

21 binding properties of the set $\left(\mathrm{K}_{d} \sim 0.15 \pm 0.04 \mathrm{nM}\right.$, Suppl. Table 2), demonstrated the

22 worst inhibitory properties (Fig. 5B). Similar to the case with the anti-PyTRAP mAb

23 panel described above, our data suggest that the PfTRAP vWA domain contains 
1 epitopes exposing vulnerability to inhibition, however lack of mAbs that strongly bind

2 other portions of PfTRAP make it difficult to discount the roles that these domains may

3 play in inhibition in vivo.

4

5 A vWA-directed anti-PfTRAP mAb increases the protection afforded by a protective

6 CSP $m A b$.

7 Because $P f$ sporozoites do not infect murine livers, the only means to test the activity of

8 anti-Pf antibodies against sporozoite infection in vivo is by either challenging passively

9 or actively immunized wild-type mice with transgenic rodent parasites expressing the $P f$

10 proteins of interest ${ }^{33-35}$ or by passive immunization of immune-deficient humanized liver

11 mice (FRG huHep), which can be challenged with $P f$ sporozoites ${ }^{14}$. We chose to utilize

12 the latter as it is an established model of antibody-mediated protection against $P f$

13 infection ${ }^{14,36-41}$ and allows testing of any future combination of anti-Pf antibodies

14 without the need for generating combinatorial transgenic parasites. In this model,

15 humanized-liver mice receive a passive transfer of antibodies and are then infected with

16 Pf via mosquito bite. Six days later, mice are injected with human red blood cells, which

17 can then be infected by emerging merozoites and infection quantified by qRT-PCR on

18 days 7 and 9.

20 Using this method, we tested the ability of the anti-PfTRAP mAb AKBR-4 to provide

21 sterile protection against $P f$ mosquito-bite infection alone or in combination with a

22 partially-protective anti-PfCSP mAb CIS43 ${ }^{37}$. We chose a dose of $50 \mu \mathrm{g} /$ mouse $(\sim 2.5$

$23 \mathrm{mg} / \mathrm{kg}$ ) for each $\mathrm{mAb}$, as this provides partial protection with an anti-PfCSP $\mathrm{mAb}{ }^{37}$ and 
1 gives a serum concentration of $\sim 10 \mu \mathrm{g} / \mathrm{mL}$ at the time of infection, which is achievable

2 by both active vaccination and passive transfer of long-lasting mAbs ${ }^{42,43}$. We previously

3 conducted passive administration, mosquito bite challenge in 2 replicate experiments ${ }^{37}$

4 that showed that $50 \mu \mathrm{g} /$ mouse dose of anti-PfCSP mAb CIS43 was protective (5/7 and

$5 \quad 5 / 8$ protected in each experiment), compared to control mice (0/7 and 0/7 protected). To

6 avoid unnecessary duplication of FRG huHep experiments, we included those cohorts

7 in our overall analysis of mAbs in this study. We conducted a third replicate experiment

8 with an additional $8 \mathrm{CIS} 43 \mathrm{mAb}$ mice and 5 control mAb mice in the current study,

9 where 5/8 CIS43 treated mice were protected and 0/5 control mice were unprotected,

10 bringing our total experimental yield to a total of $15 / 23$ protected (65\%), which was

11 significant compared to 0/19 of control mice protected $(0 \%, p<0.0001$; Table 2, Fig.

12 5C). This protection was not improved in a single experiment of 5 FRG huHep mice in

13 which the dose was increased 3-fold to $150 \mu \mathrm{g} /$ mouse $(3 / 5,60 \%$ protected; $p=0.002$

14 over control).

16 On its own, passive administration of $50 \mu \mathrm{g} / \mathrm{mouse}$ of AKBR-4 failed to provide any

17 sterile protection (0/11, 0\%). Yet, when $50 \mu \mathrm{g} /$ mouse of AKBR-4 was combined with 50

$18 \mu \mathrm{g} /$ mouse of the anti-PfCSP mAb (100 $\mu \mathrm{g} \mathrm{mAb/mouse} \mathrm{total),} \mathrm{14/16} \mathrm{(88 \% )} \mathrm{mice} \mathrm{were}$

19 sterilely protected. The improvement afforded by the AKBR-4/anti-PfCSP mAb

20 combination over the efficacy of the anti-PfCSP mAb alone (up to $65 \%$ protection with

21 up to $150 \mu \mathrm{g} /$ mouse) trended toward, but did not reach statistical significance at this

22 group size $(p=0.131)$, despite still being statistically protective compared to the control

23 antibody treatment $(p<0.0001)$. Nevertheless, together these results strongly support 
1 proof of concept that polyclonal and monoclonal antibodies directed against PfTRAP

2 can reduce Pf sporozoite invasion and traversal of hepatocytes in vitro and are able to

3 raise the protective capacity of anti-PfCSP antibodies above the threshold of $75 \%$

4 protection. 


\section{Discussion}

2 Here, we have shown that combining antibodies recognizing TRAP and CSP resulted in

3 an additive protective effect when assessed by a mosquito-bite challenge. To date, the

4 most extensive and clear proof-of-concept that non-CSP antigens can be combined with

5 CSP to provide superior sterile protection was from a recently published rodent malaria

6 study ${ }^{44}$. The protective contributions of antibodies in that study were not specifically

7 investigated, as antibodies were deemed irrelevant for protection compared to the role

8 of T cells. This was determined in the context of a vectored vaccine immunization,

9 which is biased towards the elicitation of T cells, and using an intravenous sporozoite

10 challenge, which has been shown to bypass the role of antibodies in the skin and

11 interstitial tissues 45,46 . Therefore, although promising, this study was not designed to

12 assess the substantial role of antibodies in protection from pre-erythrocytic infection as

13 has been previously reported ${ }^{45-47}$. However, these findings, along with our data,

14 suggest that enhanced protection over CSP-alone immunization is possible by way of

15 multivalent subunit vaccination targeting distinct sporozoite surface-exposed proteins.

16 Thus, further efforts should be directed at identifying additional immunogen

17 combinations in preclinical studies.

19 In light of the complex life cycle of Plasmodium, dissection of antibody function requires

20 the use of polyclonal or monoclonal antibodies in vitro or in passive transfer in vivo

21 followed by a mosquito-bite or intradermal sporozoite challenge. The challenge mode

22 may be particularly important to take advantage of the protective effects elicited by

23 antibodies while the parasite is traversing the skin ${ }^{46}$. In a further complication, studies 
1 with CSP have shown that within a functional polyclonal antibody response, ineffective

2 antibodies may make up the majority and only a subset are potent antibodies

3 distinguished by nuanced binding properties ${ }^{36,37,48-53}$. Understanding these complex

4 activities can identify superior mAb products and vaccine immunogens, yet such studies

5 have not been previously performed for TRAP or other non-CSP pre-erythrocytic

6 antibody targets. Here, we show that the polyclonal antibody response to full-length

7 PyTRAP can substantially reduce parasite infection of hepatocytes in vitro and in vivo.

8 We confirmed these observations using monoclonal antibodies and conclude that this

9 effect is likely driven by vWA and TSR-specific antibodies. These findings clearly

10 support TRAP as a functional antibody target and is in line with previous studies using

11 antibodies against TRAP protein fragments ${ }^{29}$, yet are in contrast to others that failed to

12 see significant inhibition ${ }^{30}$. Our data with PfTRAP were more limited but the only mAb

13 that was functional in vitro recognized the vWA domain. The difference in the

14 proportions of functional antibodies we observed between PyTRAP and PfTRAP may

15 be due to subtle differences in protein localization ${ }^{54,55}$ and, potentially, binding partners

1656 between the two species.

18 Achievable and minimal functional antibody levels are important parameters in vaccine 19 and monoclonal antibody product development. In our in vivo Py inhibition studies, the

20 combination of mAbs brought the total antibody dose to four times that of the anti-

21 PyCSP antibody alone $(100 \mu \mathrm{g} /$ mouse of anti-PyCSP alone to $400 \mu \mathrm{g} / \mathrm{mouse}$ total with

22 the combination). It is reasonable to expect that simply increasing the dose of the anti-

23 CSP mAb would have also yielded higher levels of protection in this mouse model. Yet 
1 experience with RTS,S—which elicits extremely high levels of anti-CSP antibodies—as

2 well as published data using highly potent anti-CSP mAbs ${ }^{37,49,57}$ suggest that

3 increasing anti-CSP titers alone provides diminishing returns. Furthermore, sustaining

4 such high levels of antibodies over years may be unachievable by vaccines or mAb

5 prophylaxis. The first CHMI trial using passive transfer of the same CIS43 anti-PfCSP

$6 \mathrm{mAb}$ used in this study indeed showed that mAbs can provide sterilizing protection

7 against $P$. falciparum mosquito bite infection at serum concentrations between $\sim 50-500$

$8 \mu \mathrm{g} / \mathrm{mL}$. However, prophylactic mAb products will likely need increased potency in order

9 to provide long-lasting protection at lower concentrations against more diverse and

10 likely more fit parasites in the field. Rather than increasing the dose or frequency of

11 administration of anti-CSP mAbs to sustain high levels of serum concentrations, long-

12 term maintenance of moderate or low titers against two or more antigens with additive

13 protection properties may be more easily achieved. Our experiments using Pf mosquito-

14 bite challenge in FRGhuHep mice support such a strategy, as we were able to achieve

15 high levels of sterile protection (88\%) using a combination of anti-PfCSP and anti-

16 PfTRAP mAbs. Importantly, this level of protection was achieved using a low total dose

17 of $\mathrm{mAb}(100 \mu \mathrm{g} / \mathrm{mouse}$ or $\sim 5 \mathrm{mg} / \mathrm{kg})$ and brought the combination above the $75-80 \%$

18 protection threshold desired for vaccines ${ }^{10}$ or injectable anti-malarials ${ }^{11}$. Furthermore,

19 our total combination dose of $100 \mu \mathrm{g} / \mathrm{mouse}(50 \mu \mathrm{g} / \mathrm{mouse}$ each of anti-PfCSP and anti-

20 PfTRAP mAb) gave a total circulating mAb concentration of $\sim 20 \mu \mathrm{g} / \mathrm{mL}-$ a level that

21 can be achieved for $\sim 36$ weeks with a single $20 \mathrm{mg} / \mathrm{kg}$ injection of long-lasting mAbs

$2242,58,59$ or $\sim 4$ years via active vaccination ${ }^{60}$. On the other hand, our data showing that

23 tripling the dose of the anti-PfCSP mAb alone $(150 \mu \mathrm{g} / \mathrm{mouse}, 7.5 \mathrm{mg} / \mathrm{kg})$ failed to 
1 improve protection, therefore, suggests that reaching desired thresholds of protection at

2 lower antibody concentrations may be more efficiently achieved by adding additional

3 antibody targets rather than increasing the anti-CSP antibody concentration.

5 In summary, we provide proof of concept that antibodies targeting TRAP can contribute

6 to sterile protection when used in combination with anti-CSP antibodies. These findings

7 support vaccine and $\mathrm{mAb}$ strategies where multiple antigens are targeted to enhance

8 the protection afforded by CSP. Specifically, we posit that rationally designed non-CSP

9 proteins or mAbs may be powerful tools for eliciting high levels of long-lasting protection

10 at lower antibody titers, as our data suggest they can enhance CSP-based protection

11 even when they do not elicit substantial sterile protection on their own. Such a

12 multivalent approach has previously been hampered by the difficulty in generating and

13 combining multiple protein-in-adjuvant formulations, but may be more easily achieved

14 by the use of mRNA-based vaccines which have proven adept as a multi-antigen

15 vaccine platform in preclinical studies ${ }^{61,62}$. Alternatively, pre-selected, multivalent mAbs

16 could be used as a cocktail, although such an approach is currently limited by cost ${ }^{63}$.

17 Our results indicate that efforts to develop a long-lasting, infection-blocking malaria

18 intervention would greatly benefit from identifying non-CSP antibody targets that

19 enhance CSP-elicited protection. 


\section{Materials and Methods}

2 Recombinant protein production

3 Recombinant proteins were produced in transiently transfected suspension culture of

4 FreeStyle 293 cells (Thermo), as previously described ${ }^{64}$. Briefly, codon-optimized

5 constructs encoding the ectodomain or deletions of Plasmodium falciparum (PfTRAP)

6 and Plasmodium yoelii TRAP (PyTRAP) were generated as fusions to the 8xHis and

$7 \quad$ AviTag ${ }^{65}$ sequences (Table 1). Following transfection using the high-density PEI

8 method ${ }^{66}$ and the subsequent 5-day incubation, cells were removed by centrifugation

9 and the culture supernatants were supplemented with $\mathrm{NaCl}(+350 \mathrm{mM})$ and sodium

10 azide (0.02\%). Treated culture supernatants were passed by gravity through NiNTA

11 agarose, washed with Wash Buffer (10 mM Tris- $\mathrm{HCl}, \mathrm{pH}$ 8, $300 \mathrm{mM} \mathrm{NaCl}, 10 \mathrm{mM}$

12 imidazole), and eluted with Elution Buffer (10 mM Tris-HCl, pH 7.4, $300 \mathrm{mM} \mathrm{NaCl,} 200$

$13 \mathrm{mM}$ imidazole). Further purification was performed by size-exclusion chromatography

14 using a calibrated Superdex 200 (10/600) column. The HIV Env gp120 control protein

15 was produced, as previously described ${ }^{67}$.

16

17 Monoclonal antibody cloning and production

18 Monoclonal antibodies were cloned and produced, as previously described ${ }^{64}$. Briefly,

19 ectodomain PfTRAP and PyTRAP constructs were used as immunogens, and their

20 biotinylated versions were used to isolate antigen-specific B cells by flow cytometry.

21 Following culture, wells containing B cells producing antigen-binding IgG were identified

22 by ELISA, immunoglobulin-encoding transcripts were amplified by RT-PCR and used for

23 the generation of heavy- and light-chain constructs for recombinant mAb expression.

24 The sequences were annotated using IgBLAST 68. 
1 To express recombinant mAbs, the plasmid DNA was used to transfect suspension

2 cultures of FreeStyle 293 cells (Thermo), as described above. After five days in culture,

3 cells were removed by centrifugation and the cultures were supplemented with $\mathrm{NaCl}$

$4 \quad(+350 \mathrm{mM})$ and sodium azide (0.02\%). Treated culture supernatants were passed by

5 gravity through Protein $\mathrm{G}$ resin equilibrated in Wash Buffer (10 mM HEPES, pH 7, 300

$6 \mathrm{mM} \mathrm{NaCl}, 2 \mathrm{mM}$ EDTA), washed with Wash Buffer, and eluted with $100 \mathrm{mM}$ glycine, pH

$7 \quad$ 2.7. Resulting eluates were buffer-exchanged by repeated centrifugal ultrafiltration with

8 HBS-E (10 mM HEPES, pH 7, 150 mM NaCl, 2 mM EDTA).

Binding kinetics measurements

11 Binding properties of mAbs were characterized using biolayer interferometry (BLI)

12 measurements on an Octet $\mathrm{QK}^{\mathrm{e}}$ instrument (Sartorius), as previously described ${ }^{64}$.

13 Briefly, antibodies in culture supernatants were immobilized on anti-Mouse IgG Fc

14 Capture biosensors and allowed to associate with antigen serially diluted (in the range

15 of $1-1000 \mathrm{nM})$ in 10x Kinetics Buffer (10xKB: PBS + 0.1\% BSA, 0.02\% Tween-20 and

$16 \quad 0.05 \%$ sodium azide) followed by dissociation in $10 x \mathrm{~KB}$. Resulting sensorgram data

17 was evaluated using ForteBio Data Analysis software (version 7.0.1.5) to generate a fit

18 to the $1: 1$ binding model and provide estimates for the $k_{o n}$ and $k_{\text {dis }}$ rate constants.

20 Coarse epitope mapping by ELISA

21 Domain specificity of the mAbs was characterized by enzyme-linked immunosorbent

22 assay (ELISA) using TRAP ectodomain and fragments from PfTRAP and PyTRAP, as

23 previously described ${ }^{64}$. 
2 Sporozoite production

3 For rodent parasite ( $P$. yoelii), female Swiss Webster (SW) mice for parasite cycles

4 were purchased from Envigo laboratories and injected intraperitoneally (i.p.) with blood

5 stage PyGFPluc ${ }^{69}$. Three days later, gametocyte exflagellation was confirmed and the

6 infected mice were used to feed female Anopheles stephensi mosquitoes. Fourteen to

$7 \quad 16$ days after the feed, salivary gland sporozoites were isolated from the mosquitoes

8 and used in mouse infections.

9 For human malaria ( $P$. falciparum) experiments, infected $A$. stephensi mosquitoes were

10 produced, as previously described ${ }^{70}$.

12 Animal studies ethics statement

13 All procedures involving animals were performed in adherence to protocols of the

14 Institutional Animal Care and Use Committee at the Seattle Children's Research

15 Institute. The study was carried out in compliance with the ARRIVE guidelines

\section{6 (https://arriveguidelines.org/).}

18 Mouse active immunization and challenge

19 To generate polyclonal serum and a source of mouse monoclonal antibodies, six to

20 eight week-old BALBc/J mice were purchased from Jackson laboratories and injected

21 intramuscularly three times at days 0, 14 and 38 using Adjuplex mixed with 20-25 $\mu \mathrm{g}$ of

22 target protein. Mice immunized with recombinant $P y$ proteins were then challenged by

23 the bite of 15 PyGFPluc-infected mosquitos, as published ${ }^{32}$. Forty-two hours later,

24 parasite liver burden was assessed by bioluminescent imaging, as previously published 
$1 \quad 32$. Mice were then immediately sacrificed and splenocytes collected and cryopreserved

2 for B cell isolation and mAb production.

4 Mice immunized with Pf proteins were immunized as above with the exception that mice

5 were additionally boosted with IV protein three days prior to sacrifice and collection and

6 cryopreservation of splenocytes.

7 For both, serum was collected from immunized mice by collecting whole blood in BD

8 microtainer serum tubes, allowing blood to clot at room temperature for at least 30

9 minutes and then centrifuged according to manufacturer's instructions to separate

10 serum for storage and use in in vitro assays.

12 Sporozoite immunofluorescence microscopy

13 Fresh-dissected Py or Pf sporozoites were stained, as previously published ${ }^{71}$. Briefly,

14 fresh-dissected Py or Pf sporozoites were fixed with 4\% PFA and air-dried onto glass

15 slides overnight. These were then permeabilized with $0.1 \%$ Triton- $X$ and stained with

16 polyclonal (serum at 1:200 dilution) or monoclonal $(10 \mu \mathrm{g} / \mathrm{mL})$ antibodies. Sporozoites

17 were identified by co-staining with either anti-MTIP or anti-CSP mAbs as well as DAPI

18 for nuclear localization. Images were acquired using an Olympus IX-70 DeltaVision

19 deconvolution microscope at 100X magnification.

21 In vitro inhibition of sporozoite traversal and invasion (ISTI)

22 In vitro ISTI was performed, as previously published for both $P y^{45}$ and $P f^{14}$. Briefly,

23 fresh-dissected sporozoites were added to hepatoma cells (Hepa1-6 for Py and HC04 
1 for $P f$ ) plated the day prior in 96-well plates in the presence of antibodies and FITC-

2 dextran in technical duplicates or triplicates. After 90 minutes, cells were fixed,

3 permeabilized and stained with fluorescently-labeled anti-CSP mAbs and analyzed by

4 flow cytometry. Invaded cells were identified by the presence of CSP and traversed

5 cells by the uptake of FITC-dextran. Within each experimental replicate, antibody-

6 treated wells were normalized to the invasion and traversal of wells treated with pre-

7 immune serum or non-specific mouse IgG which was set to $100 \%$.

9 Py passive transfer studies

10 Six to eight week-old BALBc/J mice were intravenously injected with indicated doses of

11 monoclonal antibody 24 hours prior to challenge by bite of 5 PyGFPluc-infected

12 mosquitos. Mice were followed up for infection by Giemsa-stained thin blood smear

13 every day from days 3-7 and every other day from days 8-14 for identification of blood

14 stage parasites. Mice in which we failed to identify parasites in 40,000 red blood cells

15 over the entire period were considered negative and sterilely protected. Control mice

16 were administered non-specific polyclonal mouse IgG at a dose equivalent to the

17 highest dose in experimental groups.

Pf passive transfer in FRG humanized liver mice

20 Mice repopulated with human hepatocytes (FRGhuHep) were purchased from Yecuris,

21 Inc. and infected with Pf via mosquito bite, as previously published ${ }^{14,36}$. Briefly,

22 indicated doses of mAb were intravenously injected into mice 24 hours prior to

23 challenge by the bite of 5 Pf-infected mosquitos. On day 6 post-infection, mice were 
1 intravenously injected with $400 \mu \mathrm{L}$ of human red blood cells and on days 7 and 9

2 peripheral blood was collected and assessed for parasites by qRT-PCR for Pf18s rRNA.

3 Any mouse with a Ct value above the no template control at either day was considered

4 positive for parasitemia.

5

$6 \quad$ Statistics

7 Statistical analyses and plotting were carried out in Prism (GraphPad) or in R (version

8 4.0.2) using packages Exact (version 2.1), ggpubr (version 0.4.0), ggstatsplot (version

$9 \quad$ 0.7.2). Statistical tests and outcomes are noted in the figure legend for each figure. For

10 all tests, a p-value of $<0.05$ was considered significant and values not specifically

11 labeled were above this threshold.

12

\section{Data availability}

14 DNA sequences encoding the mAbs described here have been deposited in GenBank

15 (accession numbers OK484322-OK484365). 


\section{Acknowledgements}

2 We would like to thank the vivarium staff at Seattle Children's Research Institute for

3 their support of animal studies. We would also like to thank Drs. Nevile Kisalu and

4 Robert Seder of the NIH VRC for their provision of monoclonal antibody CIS43. This

5 study was funded by NIH R01 Al117234 to DNS and SHK. 


\section{Author contributions}

2 B.K.W. and V.V. contributed equally to this work.

3 Conceptualization and experimental design: B.K.W., V.V., S.H.I.K. and D.N.S.

4 Investigation: B.K.W., V.V., S.C., N.M., N.H., A.R., H.C, B.G.O., O.T., S.K., N.D., S.A.A.

5 and N.C.

6 Data analysis and visualization: B.K.W., V.V., N.H., N.M.

7 Writing - Original draft: B.K.W.

8 Writing - Review and editing: B.K.W., V.V., S.H.I.K. and D.N.S.

9 Resources: S.H.I.K. and D.N.S.

10 Supervision, Project Administration and Funding Acquisition: B.K.W., S.H.I.K., D.N.S. 
bioRxiv preprint doi: https://doi.org/10.1101/2021.10.15.464611; this version posted October 16, 2021. The copyright holder for this preprint (which was not certified by peer review) is the author/funder, who has granted bioRxiv a license to display the preprint in perpetuity. It is made available under aCC-BY 4.0 International license.

\section{Competing Interests statement}

2 The authors declare no competing interests. 


\section{$\underline{\text { References }}$}

1. Geneva: World Health Organization. World malaria report 2020: 20 years of global progress and challenges. (2020).

2. Medica, D. L. \& Sinnis, P. Quantitative dynamics of Plasmodium yoelii sporozoite transmission by infected anopheline mosquitoes. Infect. Immun. 73, 4363-4369 (2005).

3. Ejigiri, I. \& Sinnis, P. Plasmodium sporozoite-host interactions from the dermis to the hepatocyte. Curr. Opin. Microbiol. 12, 401-407 (2009).

4. Vaughan, A. M. \& Kappe, S. H. I. Malaria Parasite Liver Infection and Exoerythrocytic Biology. Cold Spring Harb. Perspect. Med. 7, (2017).

5. Cowman, A. F., Healer, J., Marapana, D. \& Marsh, K. Malaria: Biology and Disease. Cell 167, 610-624 (2016).

6. Lindner, S. E., Miller, J. L. \& Kappe, S. H. I. Malaria parasite pre-erythrocytic infection: preparation meets opportunity. Cell. Microbiol. 14, 316-324 (2012).

7. Duffy, P. E. \& Patrick Gorres, J. Malaria vaccines since 2000: progress, priorities, products. NPJ Vaccines 5, 48 (2020).

8. Tinto, $\mathrm{H}$. et al. Long-term incidence of severe malaria following RTS,S/AS01 vaccination in children and infants in Africa: an open-label 3-year extension study of a phase 3 randomised controlled trial. Lancet Infect. Dis. 19, 821-832 (2019).

9. Epstein, J. E. et al. Protection against Plasmodium falciparum malaria by PfSPZ Vaccine. JCI Insight 2, e89154 (2017).

10. Malaria Vaccine Funders Group. Malaria Vaccine Technology Roadmap. https://www.who.int/publications/m/item/malaria-vaccine-technology-roadmap (2013).

11. Macintyre, F. et al. Injectable anti-malarials revisited: discovery and development of new agents to protect against malaria. Malar. J. 17, 402 (2018).

12. Datoo, M. S. et al. Efficacy of a low-dose candidate malaria vaccine, R21 in adjuvant Matrix-M, with seasonal administration to children in Burkina Faso: a randomised controlled trial. Lancet 397, 1809-1818 (2021).

13. Roth, A. et al. A comprehensive model for assessment of liver stage therapies targeting Plasmodium vivax and Plasmodium falciparum. Nat. Commun. 9, 1837 (2018).

14. Sack, B. K. et al. Humoral protection against mosquito bite-transmitted Plasmodium falciparum infection in humanized mice. NPJ Vaccines 2, 27 (2017).

15. Steel, R. W. J. et al. An Opsonic Phagocytosis Assay for Plasmodium falciparum Sporozoites. Clin. Vaccine Immunol. 24, (2017).

16. Boyle, M. J. et al. Human antibodies fix complement to inhibit Plasmodium falciparum invasion of erythrocytes and are associated with protection against malaria. Immunity $\mathbf{4 2}$, 580-590 (2015).

17. Swearingen, K. E. et al. Proteogenomic analysis of the total and surface-exposed proteomes of Plasmodium vivax salivary gland sporozoites. PLoS Negl. Trop. Dis. 11, e0005791 (2017).

18. Swearingen, K. E. et al. Interrogating the Plasmodium Sporozoite Surface: Identification of Surface-Exposed Proteins and Demonstration of Glycosylation on CSP and TRAP by Mass Spectrometry-Based Proteomics. PLoS Pathog. 12, (2016).

19. Lindner, S. E. et al. Total and putative surface proteomics of malaria parasite salivary gland sporozoites. Mol. Cell. Proteomics 12, 1127-1143 (2013).

20. Sack, B., Kappe, S. H. I. \& Sather, D. N. Towards functional antibody-based vaccines to prevent pre-erythrocytic malaria infection. Expert Rev. Vaccines 16, 403-414 (2017).

21. Dolo, A. et al. Thrombospondin related adhesive protein (TRAP), a potential malaria vaccine candidate. Parassitologia 41, 425-428 (1999).

22. John, C. C. et al. Correlation of high levels of antibodies to multiple pre-erythrocytic 
Plasmodium falciparum antigens and protection from infection. Am. J. Trop. Med. Hyg. 73, 222-228 (2005).

23. Hodgson, S. H. et al. Evaluation of the efficacy of ChAd63-MVA vectored vaccines expressing circumsporozoite protein and ME-TRAP against controlled human malaria infection in malaria-naive individuals. J. Infect. Dis. 211, 1076-1086 (2015).

24. Ewer, K. J. et al. Protective CD8+ T-cell immunity to human malaria induced by chimpanzee adenovirus-MVA immunisation. Nat. Commun. 4, 2836 (2013).

25. Ogwang, C. et al. Prime-boost vaccination with chimpanzee adenovirus and modified vaccinia Ankara encoding TRAP provides partial protection against Plasmodium falciparum infection in Kenyan adults. Sci. Transl. Med. 7, 286re5 (2015).

26. Gola, A. et al. Prime and target immunization protects against liver-stage malaria in mice. Sci. Transl. Med. 10, (2018).

27. Kester, K. E. et al. Sequential Phase 1 and Phase 2 randomized, controlled trials of the safety, immunogenicity and efficacy of combined pre-erythrocytic vaccine antigens RTS,S and TRAP formulated with AS02 Adjuvant System in healthy, malaria naïve adults. Vaccine 32, 6683-6691 (2014).

28. Lu, C. et al. Design and assessment of TRAP-CSP fusion antigens as effective malaria vaccines. PLoS One 15, e0216260 (2020).

29. Charoenvit, Y. et al. Development of two monoclonal antibodies against Plasmodium falciparum sporozoite surface protein 2 and mapping of B-cell epitopes. Infect. Immun. 65, 3430-3437 (1997).

30. Gantt, S. et al. Antibodies against thrombospondin-related anonymous protein do not inhibit Plasmodium sporozoite infectivity in vivo. Infect. Immun. 68, 3667-3673 (2000).

31. Rampling, T. et al. Safety and High Level Efficacy of the Combination Malaria Vaccine Regimen of RTS,S/AS01B With Chimpanzee Adenovirus 63 and Modified Vaccinia Ankara Vectored Vaccines Expressing ME-TRAP. J. Infect. Dis. 214, 772-781 (2016).

32. Sack, B. K. et al. Model for in vivo assessment of humoral protection against malaria sporozoite challenge by passive transfer of monoclonal antibodies and immune serum. Infect. Immun. 82, 808-817 (2014).

33. Atcheson, E. et al. Tailoring a Plasmodium vivax Vaccine To Enhance Efficacy through a Combination of a CSP Virus-Like Particle and TRAP Viral Vectors. Infect. Immun. 86, (2018).

34. Longley, R. J. et al. Assessment of the Plasmodium falciparum Preerythrocytic Antigen UIS3 as a Potential Candidate for a Malaria Vaccine. Infect. Immun. 85, (2017).

35. Longley, R. J. et al. Comparative assessment of vaccine vectors encoding ten malaria antigens identifies two protective liver-stage candidates. Sci. Rep. 5, 11820 (2015).

36. Tan, J. et al. A public antibody lineage that potently inhibits malaria infection through dual binding to the circumsporozoite protein. Nat. Med. 24, 401-407 (2018).

37. Kisalu, N. K. et al. A human monoclonal antibody prevents malaria infection by targeting a new site of vulnerability on the parasite. Nat. Med. 24, 408-416 (2018).

38. Triller, G. et al. Natural Parasite Exposure Induces Protective Human Anti-Malarial Antibodies. Immunity 47, 1197-1209.e10 (2017).

39. Kublin, J. G. et al. Complete attenuation of genetically engineered Plasmodium falciparum sporozoites in human subjects. Sci. Transl. Med. 9, (2017).

40. Ishizuka, A. S. et al. Protection against malaria at 1 year and immune correlates following PfSPZ vaccination. Nat. Med. 22, 614-623 (2016).

41. Foquet, L. et al. Vaccine-induced monoclonal antibodies targeting circumsporozoite protein prevent Plasmodium falciparum infection. J. Clin. Invest. 124, 140-144 (2014).

42. Gaudinski, M. R. et al. Safety and pharmacokinetics of the Fc-modified HIV-1 human monoclonal antibody VRC01LS: A Phase 1 open-label clinical trial in healthy adults. PLoS Med. 15, e1002493 (2018). 
43. Ledgerwood, J. E. et al. Safety, pharmacokinetics and neutralization of the broadly neutralizing HIV-1 human monoclonal antibody VRC01 in healthy adults. Clin. Exp. Immunol. 182, 289-301 (2015).

44. Daniel, S. et al. Plasmodium pre-erythrocytic vaccine antigens enhance sterile protection in mice induced by circumsporozoite protein. Infect. Immun. IAI0016521 (2021).

45. Keitany, G. J. et al. Immunization of mice with live-attenuated late liver stage-arresting Plasmodium yoelii parasites generates protective antibody responses to preerythrocytic stages of malaria. Infect. Immun. 82, 5143-5153 (2014).

46. Flores-Garcia, Y. et al. Antibody-Mediated Protection against Plasmodium Sporozoites Begins at the Dermal Inoculation Site. MBio 9, (2018).

47. Dups, J. N., Pepper, M. \& Cockburn, I. A. Antibody and B cell responses to Plasmodium sporozoites. Front. Microbiol. 5, 625 (2014).

48. Alanine, D. G. W. et al. Human Antibodies that Slow Erythrocyte Invasion Potentiate Malaria-Neutralizing Antibodies. Cell 178, 216-228.e21 (2019).

49. Wang, L. T. et al. A Potent Anti-Malarial Human Monoclonal Antibody Targets Circumsporozoite Protein Minor Repeats and Neutralizes Sporozoites in the Liver. Immunity (2020) doi:10.1016/j.immuni.2020.08.014.

50. Livingstone, M. C. et al. In vitro and in vivo inhibition of malaria parasite infection by monoclonal antibodies against Plasmodium falciparum circumsporozoite protein (CSP). Sci. Rep. 11, 5318 (2021).

51. Douglas, A. D. et al. A defined mechanistic correlate of protection against Plasmodium falciparum malaria in non-human primates. Nat. Commun. 10, 1953 (2019).

52. Scally, S. W. et al. Rare PfCSP C-terminal antibodies induced by live sporozoite vaccination are ineffective against malaria infection. J. Exp. Med. 215, 63-75 (2018).

53. Vijayan, K. et al. Antibody interference by a non-neutralizing antibody abrogates humoral protection against Plasmodium yoelii liver stage. Cell Rep. 36, 109489 (2021).

54. Rogers, W. O., Rogers, M. D., Hedstrom, R. C. \& Hoffman, S. L. Characterization of the gene encoding sporozoite surface protein 2, a protective Plasmodium yoelii sporozoite antigen. Mol. Biochem. Parasitol. 53, 45-51 (1992).

55. Rogers, W. O. et al. Characterization of Plasmodium falciparum sporozoite surface protein 2. Proc. Natl. Acad. Sci. U. S. A. 89, 9176-9180 (1992).

56. Steel, R. W. J. et al. Platelet derived growth factor receptor $\beta$ (PDGFR $\beta$ ) is a host receptor for the human malaria parasite adhesin TRAP. Sci. Rep. 11, 11328 (2021).

57. Kisalu, N. K. et al. Enhancing durability of CIS43 monoclonal antibody by Fc mutation or AAV delivery for malaria prevention. JCI Insight 6, (2021).

58. Gaudinski, M. R. et al. Safety and pharmacokinetics of broadly neutralising human monoclonal antibody VRC07-523LS in healthy adults: a phase 1 dose-escalation clinical trial. Lancet HIV 6, e667-e679 (2019).

59. Gaudinski, M. R. et al. A Monoclonal Antibody for Malaria Prevention. N. Engl. J. Med. 385 , 803-814 (2021).

60. Minassian, A. M. et al. Reduced blood-stage malaria growth and immune correlates in humans following RH5 vaccination. Med (N Y) 2, 701-719.e19 (2021).

61. Chahal, J. S. et al. Dendrimer-RNA nanoparticles generate protective immunity against lethal Ebola, H1N1 influenza, and Toxoplasma gondii challenges with a single dose. Proc. Natl. Acad. Sci. U. S. A. 113, E4133-42 (2016).

62. Vogel, A. B. et al. Self-Amplifying RNA Vaccines Give Equivalent Protection against Influenza to mRNA Vaccines but at Much Lower Doses. Mol. Ther. 26, 446-455 (2018).

63. Pelfrene, E., Mura, M., Cavaleiro Sanches, A. \& Cavaleri, M. Monoclonal antibodies as antiinfective products: a promising future? Clin. Microbiol. Infect. 25, 60-64 (2019).

64. Carbonetti, S. et al. A method for the isolation and characterization of functional murine monoclonal antibodies by single B cell cloning. J. Immunol. Methods 448, 66-73 (2017). 
65. Fairhead, M. \& Howarth, M. Site-specific biotinylation of purified proteins using BirA. Methods Mol. Biol. 1266, 171-184 (2015).

66. Backliwal, G., Hildinger, M., Hasija, V. \& Wurm, F. M. High-density transfection with HEK293 cells allows doubling of transient titers and removes need for a priori DNA complex formation with PEI. Biotechnol. Bioeng. 99, 721-727 (2008).

67. Sather, D. N. et al. Factors associated with the development of cross-reactive neutralizing antibodies during human immunodeficiency virus type 1 infection. J. Virol. 83, 757-769 (2009).

68. Ye, J., Ma, N., Madden, T. L. \& Ostell, J. M. IgBLAST: an immunoglobulin variable domain sequence analysis tool. Nucleic Acids Res. 41, W34-40 (2013).

69. Miller, J. L. et al. Quantitative bioluminescent imaging of pre-erythrocytic malaria parasite infection using luciferase-expressing Plasmodium yoelii. PLoS One 8, e60820 (2013).

70. Vaughan, A. M. et al. A transgenic Plasmodium falciparum NF54 strain that expresses GFP-luciferase throughout the parasite life cycle. Mol. Biochem. Parasitol. 186, 143-147 (2012).

71. Sack, B. K. et al. Mechanisms of stage-transcending protection following immunization of mice with late liver stage-arresting genetically attenuated malaria parasites. PLoS Pathog. 11, e1004855 (2015). 


\section{Tables}

2 Table 1. Combination of anti-PyCSP and anti-PyTRAP can improve sterile protection 3 from mosquito bite challenge.

4

\begin{tabular}{|c|c|c|c|c|c|c|}
\hline & \multicolumn{4}{|c|}{ Sterile Protection } & \multicolumn{2}{c|}{ Comparison p value } \\
\hline & Exp 1 & Exp 2 & Exp 3 & Combined & vs. mlgG & mlgG p value \\
\hline mlgG & $1 / 5(20 \%)$ & $0 / 5(0 \%)$ & $1 / 5(20 \%)$ & $2 / 15(13.3 \%)$ & - & 0.056 \\
\hline$\alpha$ CSP + mlgG & $4 / 8(50 \%)$ & $3 / 5(60 \%)$ & $2 / 5(40 \%)$ & $9 / 18(50 \%)$ & $\mathbf{0 . 0 5 6}$ & - \\
\hline TY11 + mlgG & $1 / 3(33 \%)$ & $0 / 5(0 \%)$ & $1 / 5(20 \%)$ & $2 / 13(15.4 \%)$ & 0.79 & 0.048 \\
\hline TY12 + mlgG & $2 / 4(50 \%)$ & $0 / 5(0 \%)$ & $0 / 4(0 \%)$ & $2 / 13(15.4 \%)$ & 0.79 & 0.048 \\
\hline TY03 + mlgG & $5 / 6(83.3 \%)$ & $0 / 5(0 \%)$ & $2 / 5(40 \%)$ & $7 / 16(43.7 \%)$ & 0.072 & 0.734 \\
\hline$\alpha C S P+$ TY11 & $2 / 4(50 \%)$ & $1 / 5(20 \%)$ & $1 / 5(20 \%)$ & $4 / 14(28.6 \%)$ & 0.355 & 0.278 \\
\hline$\alpha C S P+$ TY12 & $3 / 4(75 \%)$ & $0 / 5(0 \%)$ & $2 / 5(40 \%)$ & $5 / 14(35.7 \%)$ & 0.211 & 0.586 \\
\hline$\alpha C S P+$ TY03 & $6 / 6(100 \%)$ & $4 / 5(90 \%)$ & $4 / 5(80 \%)$ & $14 / 16(87.5 \%)$ & $\mathbf{0 . 0 0 0 0 3 4}$ & $\mathbf{0 . 0 2 5}$ \\
\hline
\end{tabular}

5
Mice were injected with $100 \mu \mathrm{g} /$ mouse of anti-CSP mAb (2F6), $300 \mu \mathrm{g} / \mathrm{mouse}$ of an anti-PyTRAP mAb or a combination of both $24 \mathrm{~h}$ prior to challenge by 5 Py-infected mosquitos. Where only one mAb was injected, mice were also given non-specific mlgG to total $400 \mu \mathrm{g} / \mathrm{mouse}$. Mice were tracked for 14 days for parasitemia by thin blood smear and those which remained parasite-free at day 14 were considered sterilely protected. Comparisons between groups were carried out via Barnard's exact test with the resulting $p$-value, as indicated. 
1 Table 2. Combination of anti-PfCSP and anti-PfTRAP can improve sterile protection

2 from mosquito bite challenge.

3

\begin{tabular}{|c|c|c|c|c|c|c|}
\hline & \multicolumn{4}{|c|}{ Sterile Protection } & \multicolumn{2}{|c|}{ Comparison $\mathrm{p}$ value } \\
\hline & Exp 1 & Exp 2 & Exp 3 & Combined & vs. mlgG & vs. $\alpha \mathrm{CSP}$ \\
\hline $150 \mu \mathrm{g} \mathrm{mlgG}$ & $0 / 7(0 \%)^{\#}$ & $0 / 7(0 \%)^{\#}$ & $0 / 5(0 \%)$ & $0 / 19(0 \%)$ & - & $<0.0001$ \\
\hline $50 \mu g$ AKBR-4 & $0 / 6(0 \%)$ & - & $0 / 5(0 \%)$ & $0 / 11(0 \%)$ & 1 & 0.0002 \\
\hline $50 \mu g \alpha C S P$ & $5 / 7(71 \%) \#$ & $5 / 8(63 \%)^{\#}$ & $5 / 8(63 \%)$ & $15 / 23(65 \%)$ & $<0.0001$ & - \\
\hline $150 \mu g$ aCSP & - & $3 / 5(60 \%)$ & - & $3 / 5(60 \%)$ & 0.002 & 0.88 \\
\hline $\begin{array}{c}50 \mu \mathrm{g} \text { AKBR-4 + } \\
50 \mu \mathrm{g} \alpha \mathrm{CSP}\end{array}$ & - & 6/7 (86\%) & $8 / 9(89 \%)$ & $14 / 16(88 \%)$ & $<0.0001$ & 0.131 \\
\hline
\end{tabular}

4

5 Mice were injected with indicated doses of either non-specific mlgG, anti-CSP mAb

6 CIS43, anti-PfTRAP mAb AKBR-4 or a combination of anti-CSP and AKBR-4 24h prior

7 to challenge with 5 Pf-infected mosquito bites. Mice were injected with human red blood

8 cells at day 5 and 6 , and then blood sampled at days 7 and 9 to detect blood stage

9 parasitemia by qPCR. Number and percentages of mice protected across 3

10 independent experiments are shown. The $p$ values for comparisons to either control

11 group (mlgG) or anti-CSP alone using Barnard's exact test are shown. \# indicates

12 results previously reported in Kisalu et al. ${ }^{49}$. 


\section{Figures}

A.
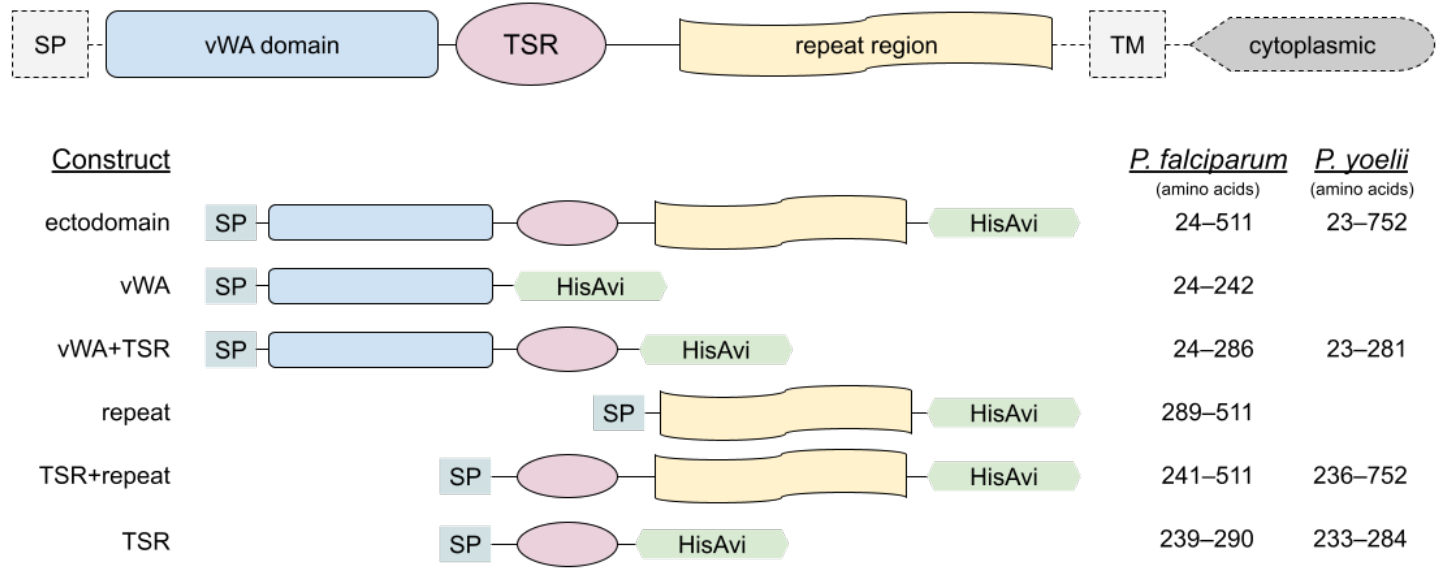

B.
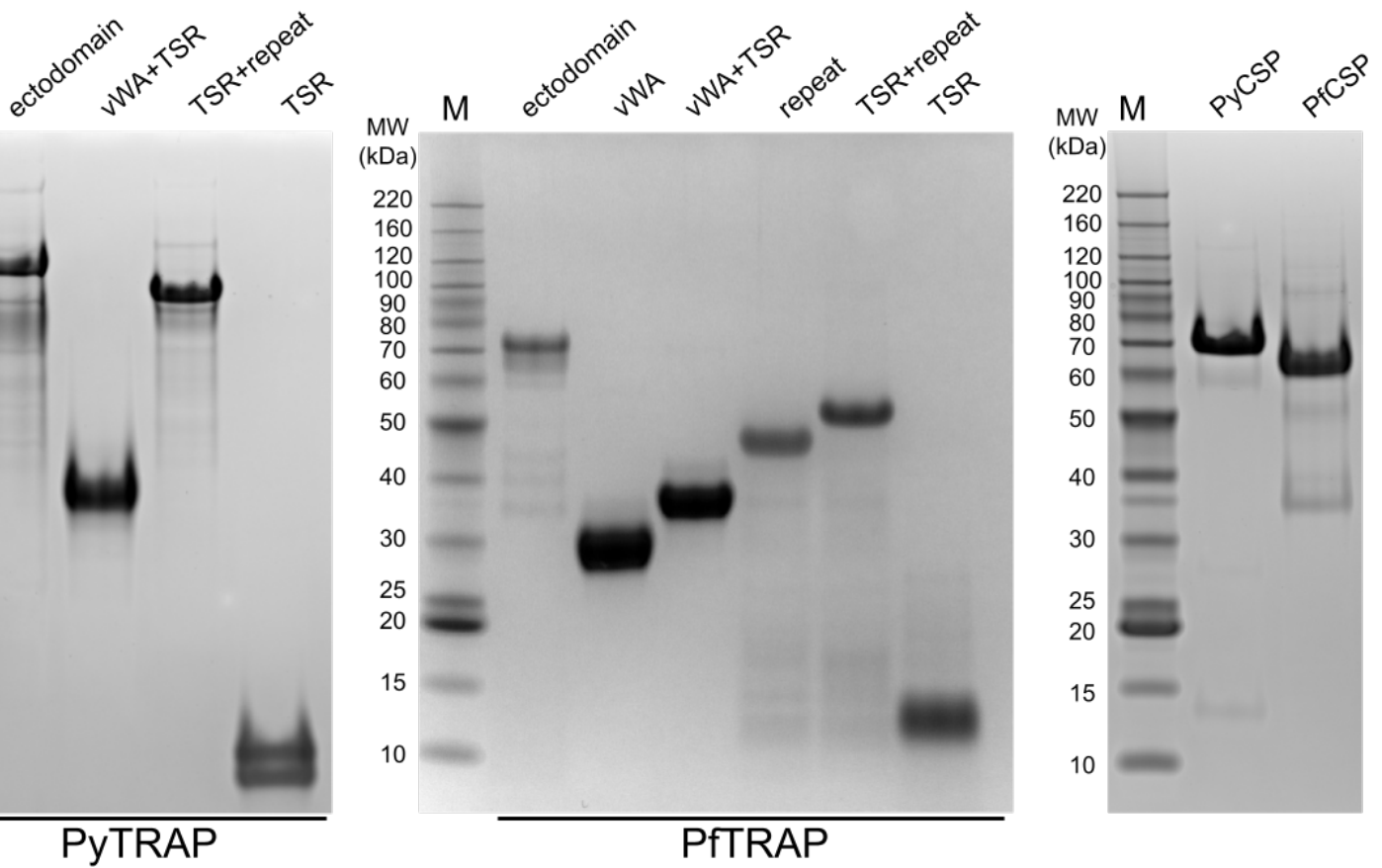

3 Figure 1. TRAP domain organization and constructs used.

4 Ectodomain and deletion constructs for PyTRAP and PfTRAP generated using the 5 domain boundaries $(\mathbf{A})$ were recombinantly expressed and purified alongside the 6 control CSP ectodomain proteins (B). 

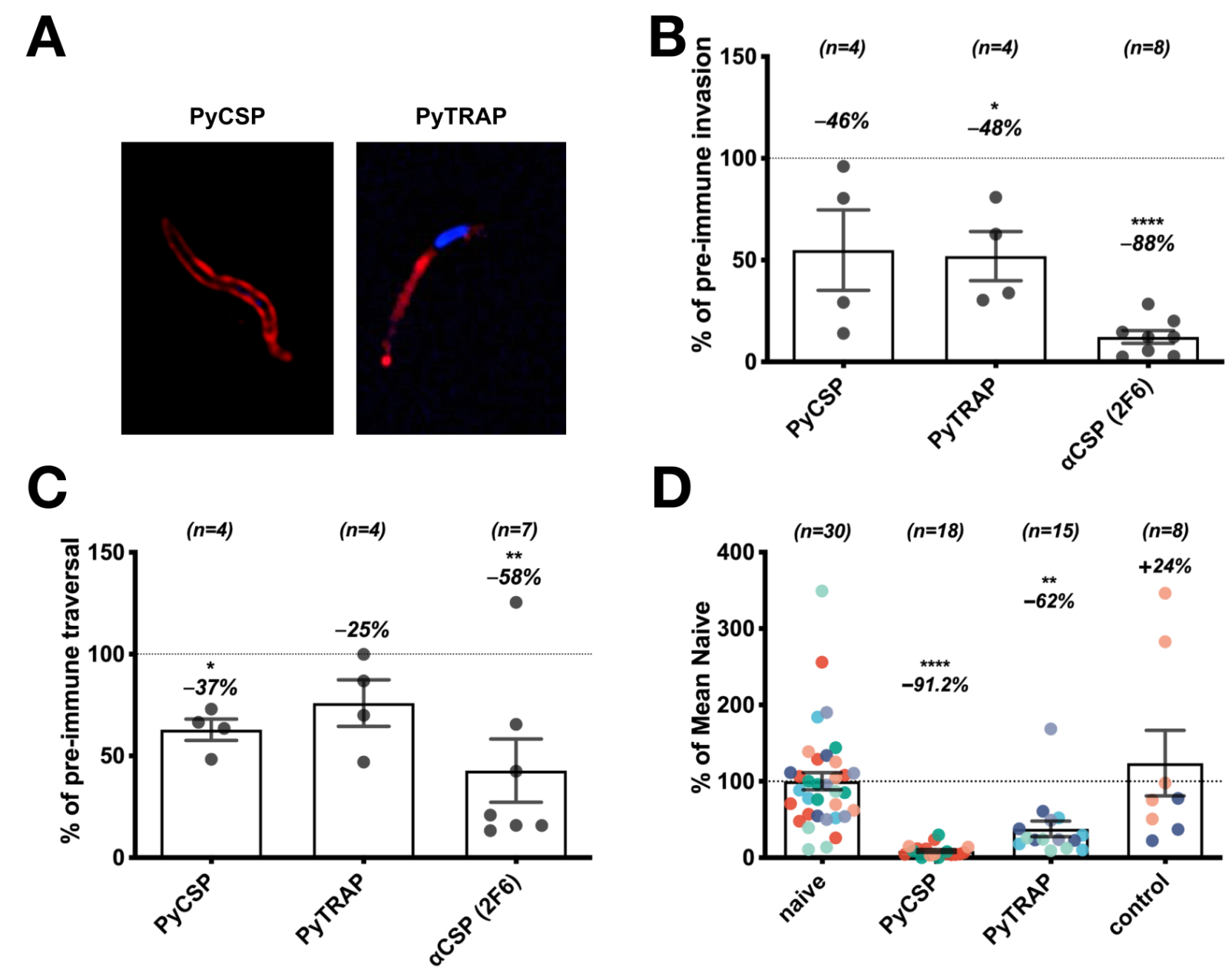

D

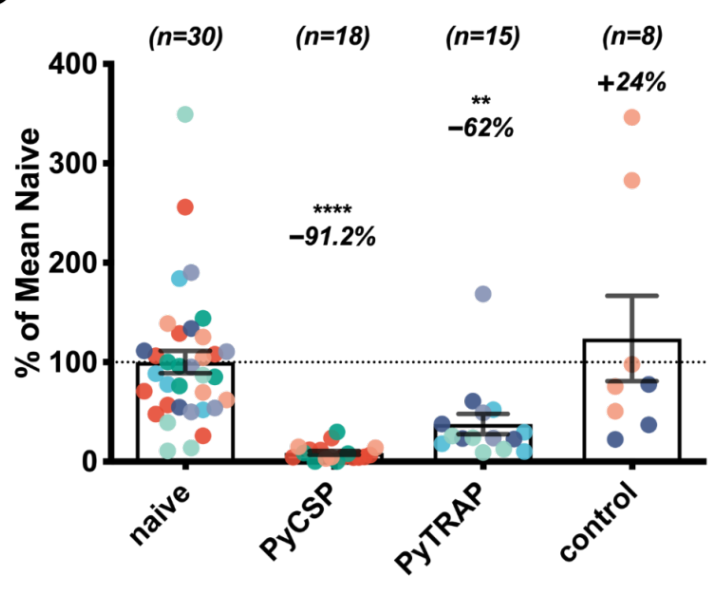

Figure 2. Polyclonal antibodies to PyTRAP inhibit parasite invasion, traversal and in vivo

infection.

Mice were immunized three times with PyTRAP and PyCSP ectodomains. A) Immune serum was used to verify binding to $P y$ sporozoite via immunofluorescence. Shown are fixed, permeabilized sporozoites labeled with a 1:800 dilution of polyclonal mouse serum followed by anti-mouse lgG (red channel) and DAPI nuclear stain (blue). Immune serum was then assessed for function in vitro for inhibition of invasion (B) and traversal (C). In B and C, each data point is the average "\% of pre-immune" invasion or traversal for pooled serum from a cohort of 5 mice across independent assays (number shown above each bar). Each bar indicates the mean "\% of pre-immune" parasite invasion or traversal with percent change from 100\% (shown as dashed line) shown above. Asterisks indicate a significant difference from $100 \%$ as determined by a two-tailed onesample $t$-test. D) Immunized mice were challenged by the bite of 15 PyGFPluc-infected mosquitos and assessed for parasite liver burden by bioluminescent imaging. Each data point represents an individual mouse with each color corresponding to an independent immunization-challenge experiment (total number of animals shown above each bar).

18 Each data point is normalized to the mean luminescent signal from "naive" mice within 
bioRxiv preprint doi: https://doi.org/10.1101/2021.10.15.464611; this version posted October 16, 2021. The copyright holder for this preprint (which was not certified by peer review) is the author/funder, who has granted bioRxiv a license to display the preprint in perpetuity. It is made available under aCC-BY 4.0 International license.

1 each challenge experiment while "control" mice are an additional group immunized with 2 HIV Env gp120 protein. Asterisks indicate significance as determined by ANOVA with

3 Kruskall-Wallis post-test. For $\mathbf{B}-\mathbf{D}$, * is $p \leq 0.05$; ${ }^{* *}$ is $p \leq 0.01$; and ${ }^{* * *}$ is $p \leq 0.0001$. 
A

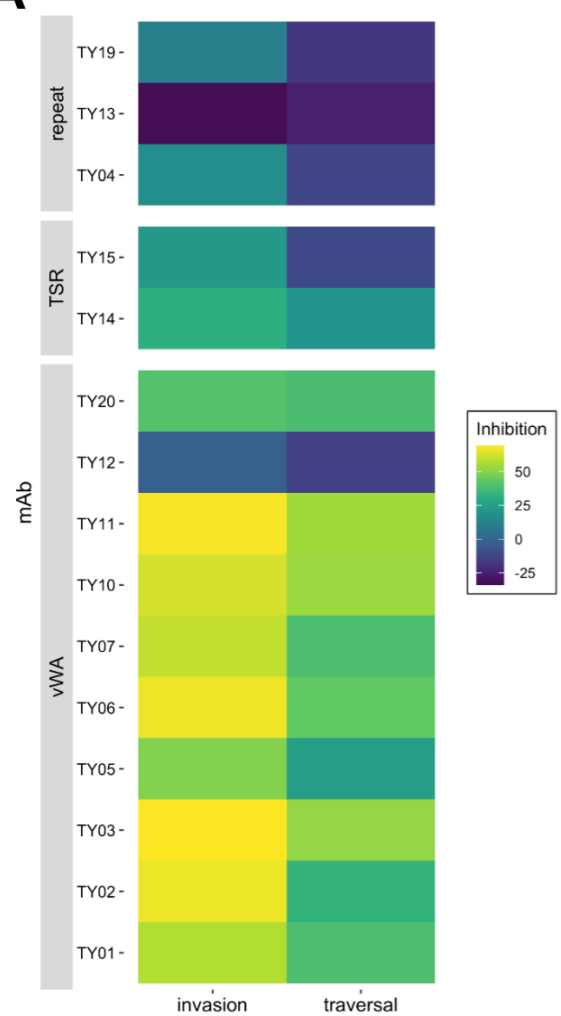

B

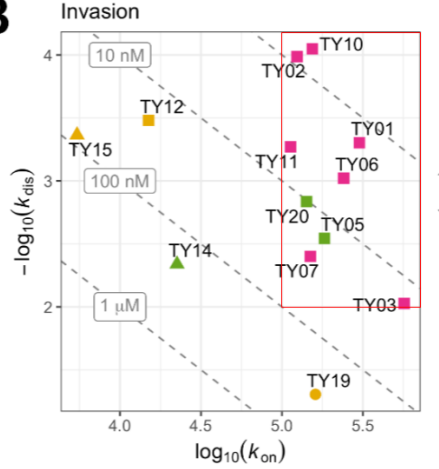

C

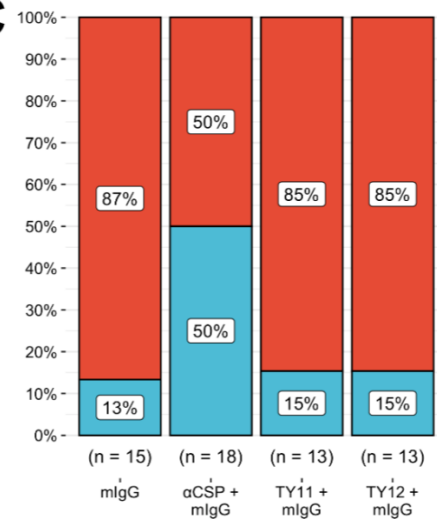

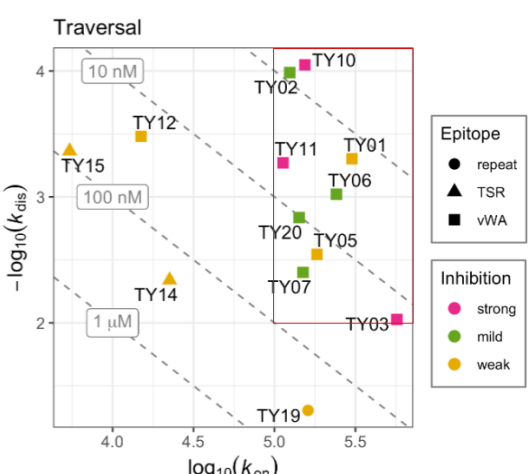

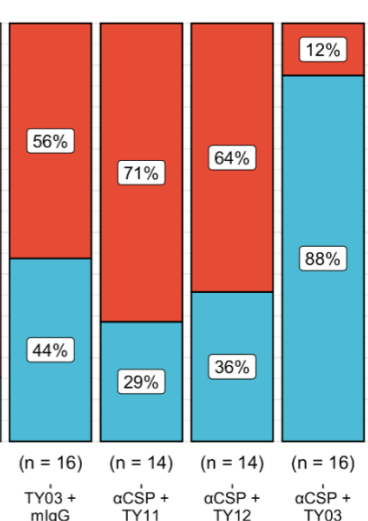

Figure 3. Effects of PyTRAP monoclonal antibodies on parasite activity.

Each $\mathrm{mAb}$ was assessed for in vitro function of inhibition of invasion and traversal (A).

4 In each case, mean values of \% inhibition (i.e., $100 \%$ - invasion or traversal value) from

5 the $100-\mu \mathrm{g} / \mathrm{mL} \mathrm{mAb}$ concentrations (bar plots with these and additional conditions

6 shown in Suppl. Fig. 1) are represented on a color axis. Binding kinetics for each mAb

7 was measured by BLI and shown as kinetic maps (B) with gray dashed diagonal

8 contour lines labeled with the corresponding $K_{d}$ values and symbols representing the

9 characterized epitopes for invasion (left) and traversal (right) inhibition. Higher-affinity

10 (i.e., those possessing lower $K_{d}$ values) mAbs are closer to the upper-right corner of this

11 plot. Symbol color coding represents "strong" inhibition for mean values $\leq 50 \%$, "mild"

12 inhibition for values $\leq 70 \%$ and "none" for mean values $>70 \%$ observed at the 100 -

$13 \mu \mathrm{g} / \mathrm{mL}$ concentration. Red box highlights the region of the kinetic plots containing the

14 values for mAbs that showed strong inhibition in invasion and traversal assays.

15 Summarized sterile protection ratios following passive-transfer-challenge experiments

16 (C; number of animals in each group is shown below the corresponding bar, individual

17 values shown in Table 1 ). 


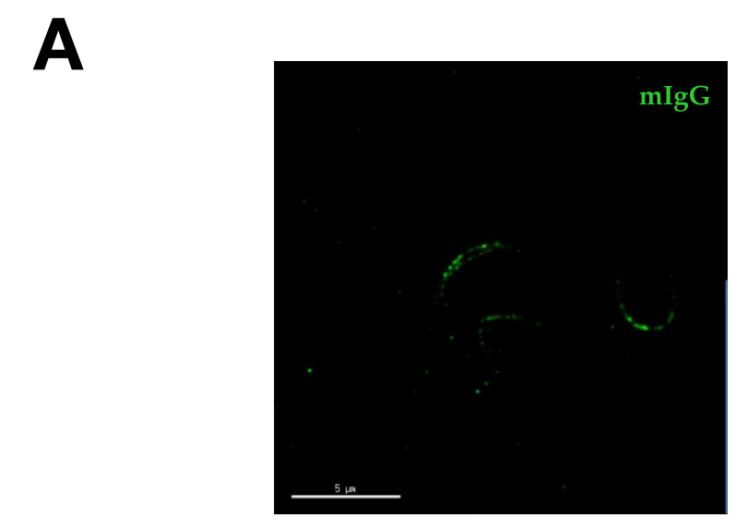

B
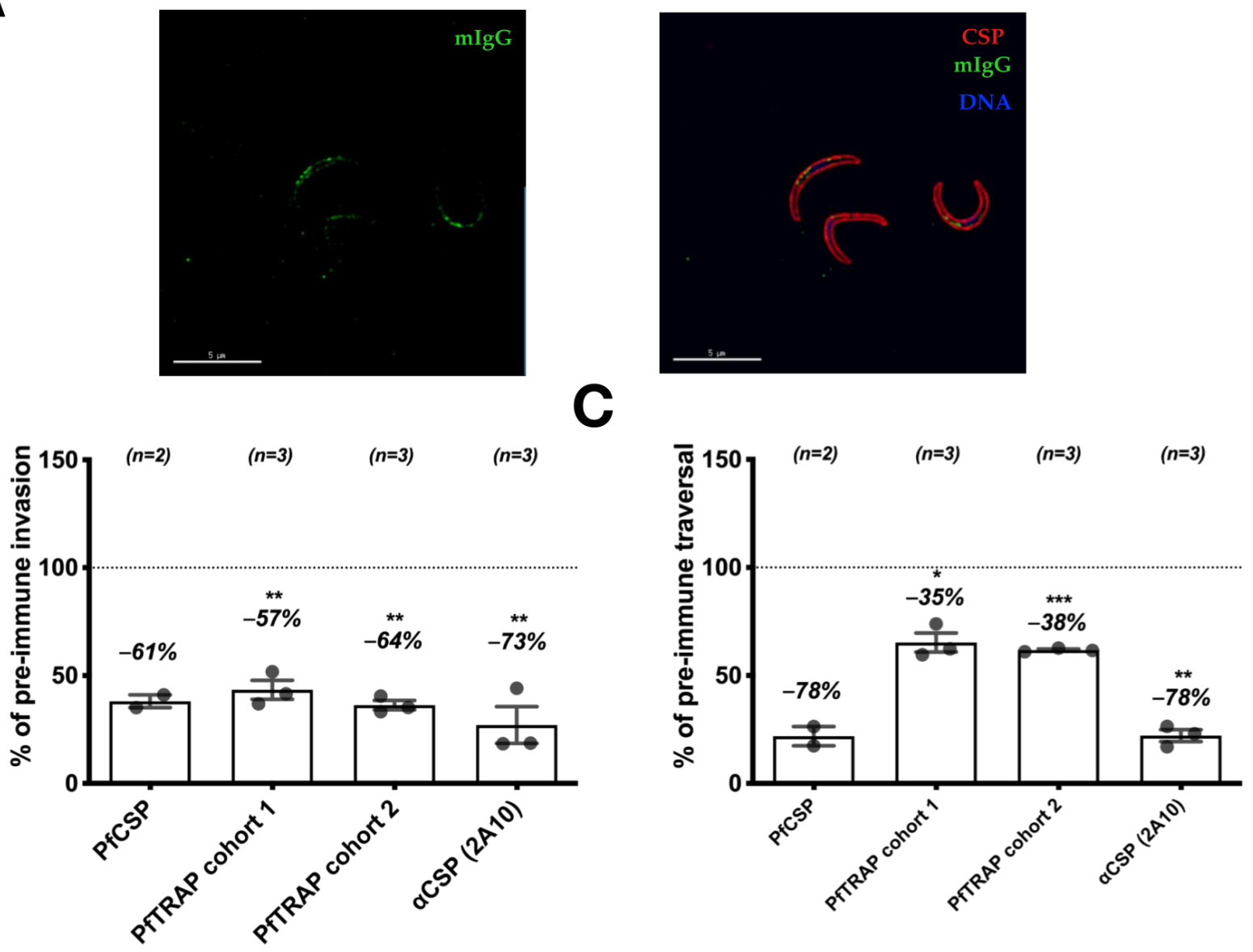

2 Figure 4. Polyclonal antibodies to PfTRAP inhibit parasite invasion, traversal in vitro.

3 Mice were immunized three times with full-length PfTRAP and PfCSP. A) Immune 4 serum was used to verify binding to $P f$ sporozoite via immunofluorescence. Shown are 5 fixed, permeabilized sporozoites labeled with a 1:800 dilution of polyclonal mouse 6 serum followed by anti-mouse IgG (green channel), anti-PfCSP monoclonal antibody $7 \quad 2 A 10$ (red channel, right image) and DAPI nuclear stain (blue, right image). Immune 8 serum was then assessed for function in vitro for inhibition of invasion (B) and traversal 9 (C). In B and C, each data point is the average \% of Pre-immune invasion or traversal 10 from technical triplicates in independent experiments. Each bar indicates the mean \% of

11 Pre-immune parasite invasion or traversal \pm SEM with exact mean above. Asterisks 12 indicate a significant difference from $100 \%$ as determined by one-way t test where ${ }^{*}$ is $13 \mathrm{p} \leq 0.05 ;{ }^{* *}$ is $\mathrm{p} \leq 0.01$; and ${ }^{* * *}$ is $\mathrm{p} \leq 0.0001$. 
A

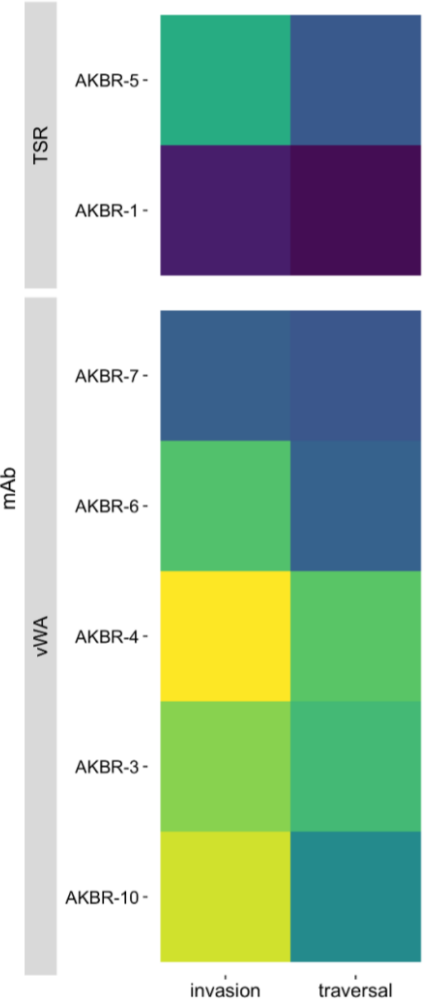

B
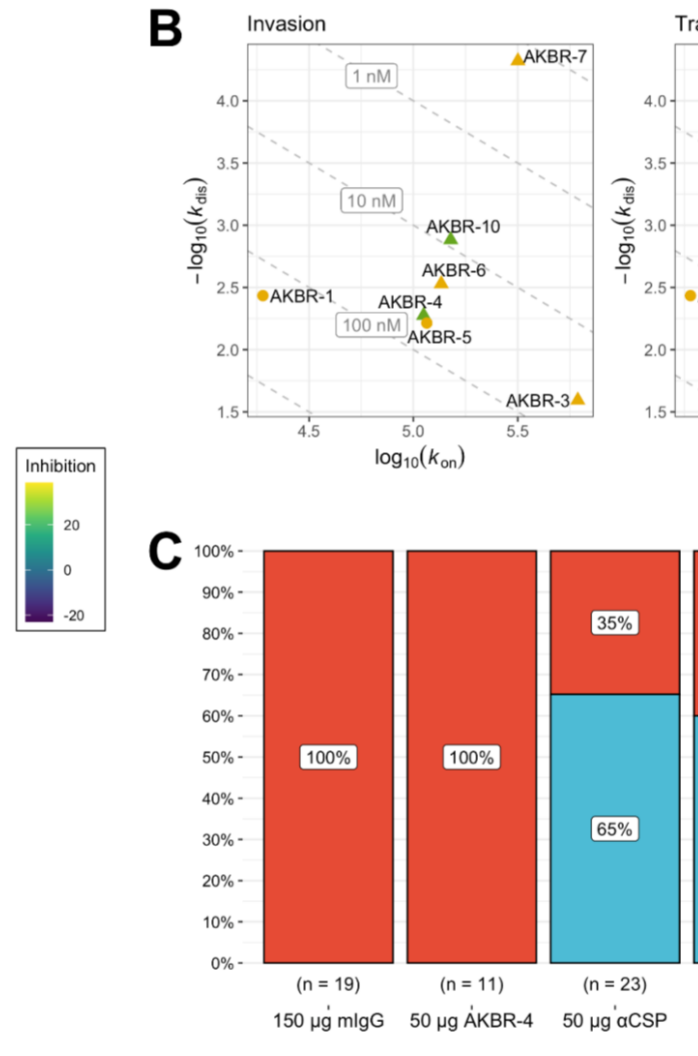
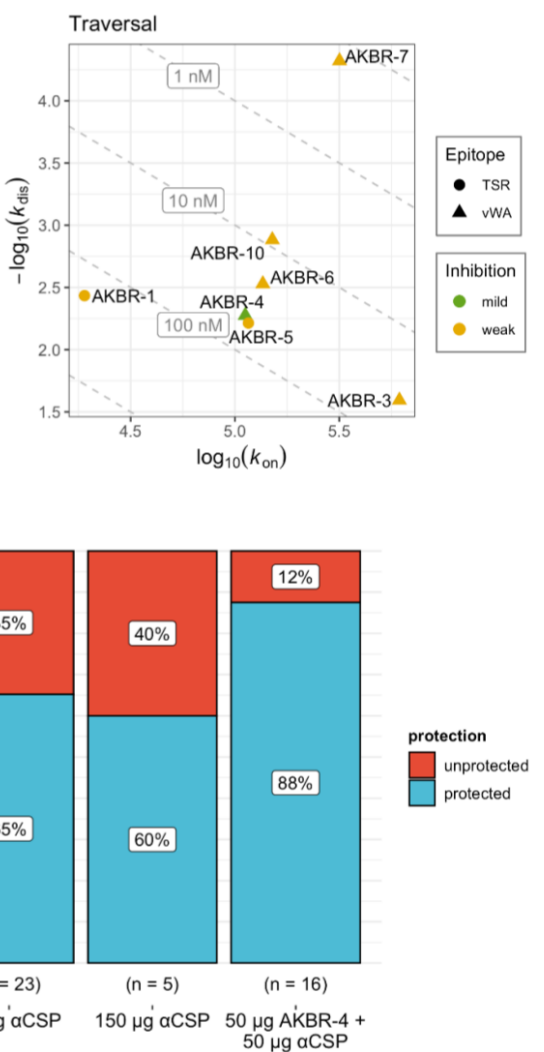

Figure 5. Monoclonal antibodies to PfTRAP inhibit parasite invasion, traversal in vitro.

Each $\mathrm{mAb}$ was assessed for in vitro function of inhibition of invasion and traversal (A). In each case, mean values of \% inhibition (i.e., $100 \%$ - invasion or traversal value) from the $100-\mu \mathrm{g} / \mathrm{mL} \mathrm{mAb}$ concentrations (bar plots with these and additional conditions shown in Suppl. Fig. 3) are represented on a color axis. Binding kinetics for each mAb was measured by $\mathrm{BLI}$ and shown as kinetic maps (B) with gray dashed diagonal contour lines labeled with the corresponding $K_{d}$ values and symbols representing the characterized epitopes for invasion (left) and traversal (right) inhibition. Higher-affinity (i.e., those possessing lower $\mathrm{K}_{d}$ values) mAbs are closer to the upper-right corner of this plot. Symbol color coding represents "strong" inhibition for mean values $\leq 50 \%$, "mild"

$13 \mu \mathrm{g} / \mathrm{mL}$ concentration. Summarized sterile protection breakdowns following passive-

14 transfer-challenge experiments ( $\mathbf{C}$; number of animals in each group is shown below the 15 corresponding bar, individual values shown in Table 2). 